K.A. van der Hucht, G. Koenigsberger \& P.R.J. Eenens, eds.

\title{
Hard X-ray-to-radio energy distributions in starburst galaxies
}

\author{
Miguel Cerviño and J. Miguel Mas-Hesse
}

LAEFF-INTA, P.O. Box 50727, E-28080 Madrid, Spain.

\begin{abstract}
We present in this contribution the predictions on the multiwavelength spectral energy distribution of our evolutionary population synthesis models including single and binary stellar systems. The high energy computations include the emission associated to X-ray binaries and supernovae remnants, as well as the mechanical energy released into the interstellar medium, which can be partially reprocessed into thermal X-rays. With these components we compute the spectral energy distribution of starburst galaxies from X-ray to radio ranges, and analyze finally the effects of the high energy emission on the $\mathrm{H}$ and He ionizing continuum.
\end{abstract}

\section{Predictions on spectral energy distribution (SED)}

Our models including the evolution of single and binary stars have been discussed in an accompanying paper (Mas-Hesse \& Cerviño, these Proceedings). Here we present the predictions corresponding to the multiwavelength SED and the He and $\mathrm{H}$ emission lines. The main contribution of this work is the inclusion of the X-ray domain in the multiwavelength energy distribution of evolutionary synthesis models. The basic components of the X-ray emission included in the code are:

- Mechanical energy released by SN and stellar winds in the ISM, which is partially reprocessed to thermal soft X-rays.

- Supernovae remnants, which have been assumed to generate two components with a composed Raymond-Smith spectrum.

- High mass X-ray binaries, with circular orbits (i.e., giant or supergiant stars) and stellar wind accretion onto a compact object, producing hard black body emission.

We show in Fig. 1 the predicted SED for two ages, assuming an instantaneous burst. A very young cluster $(0.25 \mathrm{Myr})$ shows thermal radio emission, some thermal soft X-ray contribution from the hot, diffuse interstellar gas and almost no emission at hard X-rays. At $16 \mathrm{Myr}$, however, the radio emission has become non-thermal and is dominated by supernova remnants. Supernova remnants also contribute to the soft X-ray emission, and a strong hard X-ray component associated to high-mass-X-ray binaries appears. The far infrared emission in the IRAS band is due to dust reprocessing thermally the absorbed UV photons. 


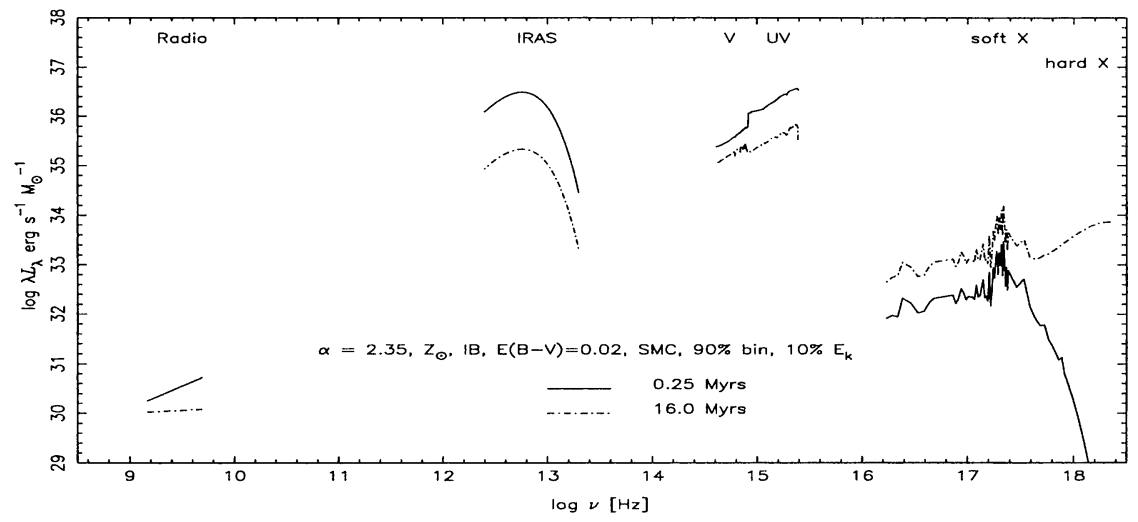

Figure 1. Multi-wavelength spectral energy distributions at two ages.

\section{The effect on the emission lines}

The effect of binary evolution on the H-emission lines is shown in Fig. 2a. It can be seen that the ionizing flux (and therefore $W(\mathrm{H} \beta)$ as well) decreases much slower than in a single-star-only cluster, due to the formation of hot WR and OB stars by mass transfer at ages above $5 \mathrm{Myr}$. On the other hand, the additional ionizing photons produced by binary systems and by the hot interstellar gas increase significantly the $L(\mathrm{He}$ II $4686 \AA) / L(\mathrm{H} \beta)$ ratio (by more than a factor 10 at ages larger than $5 \mathrm{Myr}$; see Fig. 2b). Assuming a high efficiency in the reprocessing of mechanical energy into soft X-rays (top line in Fig. 2b), it is possible to reproduce the observational values of this ratio, which shows an average around $L(\mathrm{He} \mathrm{II}) / L(\mathrm{H} \beta) \approx 0.01$. We conclude that this additional component of the ionizing flux might be (partially) responsible of the relatively large $L(\mathrm{HeII}) / L(\mathrm{H} \beta)$ values observed in several starburst galaxies.
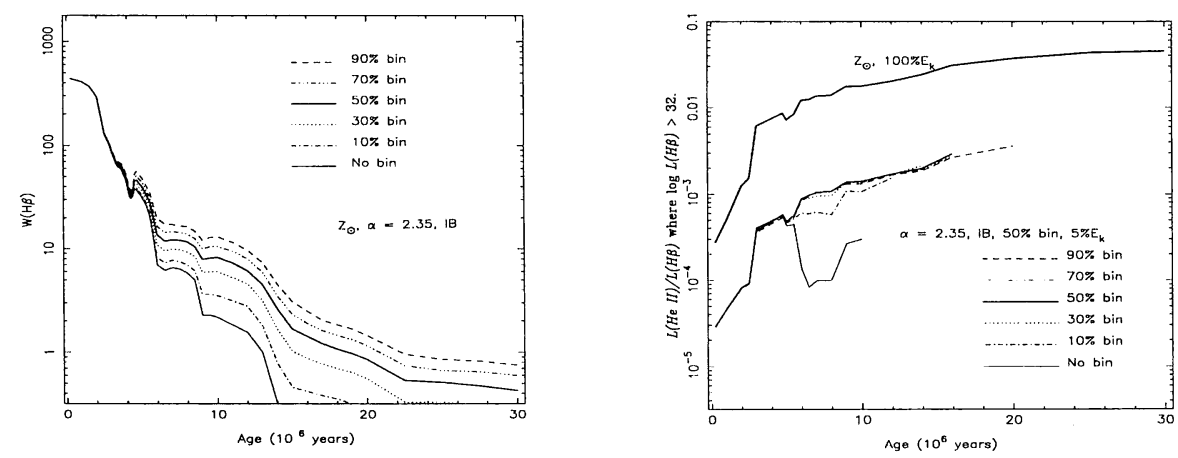

Figure 2. $\quad E W(\mathrm{H} \beta)$ and $L(\mathrm{HeII}) / L(\mathrm{H} \beta)$ as a function of the frequency of binary systems. The top line in (b) has been computed assuming a $100 \%$ efficiency in the conversion of mechanical energy into soft X-rays, which contribute to the ionization of HeII. 